\title{
Systematic review of nutritional interventions to prevent healthcare-associated infections in undernourished elderly
}

\author{
A. Bak ${ }^{1}$, J. Browne ${ }^{1}$, H. P. Loveday ${ }^{1}$ and A. Tsiami ${ }^{1}$ \\ ${ }^{1}$ Joanna Briggs Institute Collaboration Centre, University of West London, Brentford TW8 9GA, UK
}

Undernutrition in elderly patients is a major public health concern. Infections could be related to malnutrition, and may have a detrimental effect on health. The recognition and treatment of undernutrition may prevent infections in elderly. This systematic review explored a link between undernourished elderly and the risk of acquiring infections in a healthcare setting. It also explored the effectiveness of nutritional interventions in this group to prevent healthcare associated infections (HCAIs).

A search was performed according to Joanna Briggs Institute systematic review guidelines ${ }^{(1)}$ was performed. Comprehensive search of key words was used to explore the following databases: MEDLINE, EMBASE, CINAHL and Cochrane Library. The search was limited to articles published in English and after 1990. The inclusion criteria were people $\geq 60$ and undernourished. The outcome was limited to HCAIs only.

The search has generated 253 papers, of which 9 were identified as meeting inclusion criteria (3 interventions ${ }^{(2-4)}$ and 6 descriptive studies $^{(5-10)}$ ) and were included in final analysis.

\begin{tabular}{|c|c|c|c|c|c|c|}
\hline Study & Type of study & Population & Groups & $N$ & No of HCAIs & No of part. with HCAIs \\
\hline \multirow{2}{*}{ Gamaletsou et al. 2012} & Prospective & \multirow{2}{*}{ Acutely ill } & Undernourished & 10 & $\mathrm{n} / \mathrm{a}$ & 31 \\
\hline & $(\operatorname{LOS} 10.3 \mathrm{~d})$ & & Well Nourished & 127 & $\mathrm{n} / \mathrm{a}$ & 25 \\
\hline Bourdel- & Prospective & \multirow[t]{2}{*}{ Critically ill } & Undernourished & 350 & 77 & $\mathrm{n} / \mathrm{a}$ \\
\hline Marchasson et al. 1999 & $(\operatorname{LOS} 18.8 \mathrm{~d})$ & & Well Nourished & 51 & 12 & $\mathrm{n} / \mathrm{a}$ \\
\hline \multirow{2}{*}{ Bouillanne et al. 2005} & Prospective & \multirow{2}{*}{ Hospitalised } & Undernourished & 121 & 50 & $\mathrm{n} / \mathrm{a}$ \\
\hline & (LOS 71.2d) & & Well Nourished & 60 & 9 & $\mathrm{n} / \mathrm{a}$ \\
\hline \multirow[t]{2}{*}{ Batsis et al. 2009} & Prospective & \multirow[t]{2}{*}{ Hip fracture } & Undernourished & 184 & 25 & $\mathrm{n} / \mathrm{a}$ \\
\hline & $(\operatorname{LOS} 24.5 \mathrm{~d})$ & & Well Nourished & 996 & 136 & $\mathrm{n} / \mathrm{a}$ \\
\hline \multirow[t]{2}{*}{ Kuikka et al. 2009} & Prospective & \multirow[t]{2}{*}{ Residents } & Undernourished & 18 & 40 & $\mathrm{n} / \mathrm{a}$ \\
\hline & (duration $8 \mathrm{M}$ ) & & Well Nourished & 36 & 47 & $\mathrm{n} / \mathrm{a}$ \\
\hline \multirow[t]{2}{*}{ Bruce et al. 1999} & Case & \multirow[t]{2}{*}{ Hip fracture } & Undernourished & 35 & $\mathrm{n} / \mathrm{a}$ & 15 \\
\hline & (LOS 11.1d) & & Well Nourished & 65 & $\mathrm{n} / \mathrm{a}$ & 29 \\
\hline Rypkema et al. 2003 & Pseudo-RCT (LOS 32.7d) & Non-terminally ill & Intervention Control & 140 & 33 & $\mathrm{n} / \mathrm{a}$ \\
\hline \multirow[t]{2}{*}{ Johansen et al. 2004} & $\mathrm{RCT}$ & \multirow[t]{2}{*}{ Hospitalized } & Intervention & 108 & $\mathrm{n} / \mathrm{a}$ & 20 \\
\hline & (LOS 9.9d) & & Control & 104 & $\mathrm{n} / \mathrm{a}$ & 12 \\
\hline \multirow[t]{2}{*}{ Aquilani et al. 2010} & $\mathrm{RCT}$ & \multirow{2}{*}{$\begin{array}{l}\text { Post-acute } \\
\text { illness }\end{array}$} & Intervention & 40 & $\mathrm{n} / \mathrm{a}$ & 21 \\
\hline & (duration 30d) & & Control & 40 & $\mathrm{n} / \mathrm{a}$ & 33 \\
\hline
\end{tabular}

Setting: hospital except Kuikka et al. (nursing home) and Aquillani et al. (Rehabilitation Centre), LOS = Mean Length Of Stay, n/a = data not available, $p$ value for all studies either unavailable or not significant.

The data presented in the studies does not show a direct link between nutrition and HCAIs. The studies however were not designed to evaluate nutrition as a factor influencing development of HCAIs. Duration, number of people and parameters reported were not consistent in these studies, resulting in a poor quality of the data.

1. Pearson et al. (2005) Int J Evid Based Healthc 3, 207-215.

2. Rypkema et al. (2004) J Nutr Health Aging 8, 122-127.

3. Johansen et al. (2004) Clin Nutr 23, 539-550.

4. Aquilani et al. (2011) Arch Gerontol Geriatr 52, 123-128.

5. Batsis et al. (2009) J Hosp Med 4, 1-9.

6. Bouillanne et al. (2005) Am J Clin Nutr 82, 777-783.

7. Bourdel-Marchasson et al. (1999) Clin Nutr 18, 233-240.

8. Bruce et al. (1999) Aust J Ageing 18, 119-123.

9. Gamaletsou et al. (2012) J Hosp Infect 80, 68-172.

10. Kuikka et al. (2009) J Am Med Dir Assoc 10, 348-353. 